\title{
Sistema de justicia juvenil en la provincia de Buenos Aires y métodos de evaluación
}

\author{
The Juvenile Justice System in the Province of \\ Buenos Aires and evaluation methods
}

Recibido: octubre 5 de 2011 | Revisado: mayo 10 de 2012 | Aceptado: junio 10 de 2012

\author{
JoRge O. Folino* \\ MARÍA J. LESCANO ** \\ Universidad Nacional de La Plata, Buenos Aires, Argentina \\ AlFONSO SÁNCHEZ-WILDE *** \\ Ministerio de Desarrollo Humano, Buenos Aires, Argentina
}

SICI: 1657-9267(201212)11:4<1065:SJJPBA>2.0.TX;2-N

Para citar este artículo: Folino, J. O., Lescano, M. J. \& Sánchez-Wilde, A. (2012). Sistema de justicia juvenil en la provincia de Buenos Aires y métodos de evaluación. Universitas Psychologica, 11(4), 1065. 1079

* Profesor de Psiquiatría, Universidad Nacional de La Plata, Argentina. Experito I del Poder Judicial de la provincia de Buenos Aires (Argentina). E-mail: jorgefolino@fibertel.com.ar. ResearcherID: Folino, J. G-8506-2012.

** Doctora en Derecho Penal, Universidad de Salamanca. Juez, Juzgado de Garantías del Joven N.o 1, La Plata. Secretaria del Instituto de Derechos del Niño de la Facultad de Ciencias Jurídicas y Sociales, Universidad Nacional de La Plata, Argentina. Email:mjoselescano@yahoo.com.ar. ResearcherID: Lescano, M. J. H-2005-2012.

**** Defensor oficial, Régimen de Responsabilidad Penal Juvenil, Poder Judicial de la provincia de Buenos Aires. Exdirector Provincial del Sistema de Responsabilidad Penal Juvenil, Ministerio de Desarrollo Humano, Provincia de Buenos Aires, Argentina. E-mail: alfonsosw@hotmail.com.ResearcherID: Sánchez-Wilde, A. G-9551-2012.

\section{RESUMEN}

Los objetivos del presente artículo son describir el sistema de justicia juvenil en la provincia de Buenos Aires (Argentina) desde las perspectivas jurídica y ejecutiva, y comunicar avances científicos globales en la evaluación de los jóvenes que cometen actos disociales. En la provincia de Buenos Aires el sistema ha sido profundamente modificado en los últimos 15 años siguiendo las premisas de la Convención Internacional sobre los Derechos del Niño y dejando relegado el modelo del Patronato, que había regido previamente. Las instituciones encargadas de la ejecución de la intervención dispuesta por el Poder Judicial dependen actualmente de la Subsecretaria de Niñez y Adolescencia del Ministerio de Desarrollo Social y constan de centros cerrados, centros con régimen de semilibertad y centros de intervención ambulatoria (medidas alternativas a la prisión, tales como suspensión de juicio a prueba o tareas comunitarias). Las ciencias relacionadas con la salud mental y con el comportamiento vienen contribuyendo a la identificación de diversos factores influyentes en la conducta disocial y con el diseño de tipos de intervención para cada nivel de prevención. Sin embargo, hay una distancia importante entre las potencialidades del estado actual del conocimiento y la implementación. En las condiciones expuestas, queda configurado un período de transición que aún requiere optimización de la política de infancia con ajustes legislativos, judiciales y administrativos y con la capitalización de los aportes científicos.

Palabras clave autores:

Justicia juvenil, conducta disocial, evaluación.

Palabras clave descriptores:

Poder Judicial, infancia, Buenos Aires.

\section{A B S T R A C T}

The aim of this article is to describe the Justice System for juveniles in the Province of Buenos Aires, Argentina, from a legal and administrative perspective; and to review recent contributions to the assessment of youth with dissocial behaviors. During the last 15 years, the system has been deeply modified in order to fit the recommendations of the Convention on the Rights of the Child's. At present, the dispositions of the Courts are carried out by the Sub - Secretariat of Childhood and Youngsters of the Ministry of Social Development. This Secretariat has different facilities, such as closed, mixed centers that combine indoors and outdoors detention centers, open centers (in charge of the application of the sentences that bear alternatives to prison dispositions and community enforced services, etc). Studies from 
mental health and behavioral sciences have contributed to the identification of risk factors for dissocial behavior and for developing adequate interventions for each specific level of prevention. However, there is still a big gap between the state of the art and the practical issues related to it. Thus, the local system can be considered to be in transition period, and in need of adjustments not only in the legal codes but also in the application of the law, if policy improvement and better use of scientific knowledge is intended.

Key words authors:

Juvenile Justice, Dissocial Behavior, Assessment.

Key words plus:

Judicial Branch, Childhood, Buenos Aires.

\section{Introducción}

En el segundo lustro de los años 2000 iniciaba su marcha un nuevo sistema de justicia juvenil en la provincia de Buenos Aires (Argentina), y también se ponía de manifiesto la escasez de investigaciones locales sobre el tema (Folino, Domenech, Gutierrez \& Lescano, 2009). A comienzos del tercer lustro, el nuevo sistema aún continúa ajustando su funcionamiento y queda pendiente perfeccionar los sistemas de evaluación y tratamiento de niños y jóvenes que cometen actos disociales.

Independientemente de la distancia con lo deseable, resulta oportuno examinar el estado actual, con la meta de contribuir al proceso de desarrollo. El presente escrito tiene por objetivo describir el sistema local desde las perspectivas jurídica y ejecutiva, y comunicar avances del área de evaluación tanto locales como internacionales.

\section{Contexto de la provincia de Buenos Aires}

Las dos instituciones bonaerenses con mayor injerencia sobre la población juvenil que comete delitos son el Poder Judicial de la Provincia de Buenos Aires y el Ministerio de Desarrollo Social. Adicionalmente, en el sistema participan instituciones privadas como clínicas y comunidades terapéuticas y otras públicas, tanto sanitarias como educativas. En todas ellas, hay inserción de profesionales de la salud mental, y oportunidades para la evaluación de los jóvenes y la planificación de intervenciones.

\section{El Poder Judicial}

El sistema de la justicia juvenil en la provincia de Buenos Aires ha sido profundamente modificado a partir de la ley $13298^{1}$ y su complementaria, la ley $13634^{2}$. Estas leyes se gestaron al amparo de las premisas emanadas por la Convención Internacional sobre los Derechos del Niño que han venido a consagrar un nuevo sistema, denominado de Promoción y Protección de los Derechos de Niños, Niñas y Adolescentes ${ }^{3}$.

La sanción de este modelo normativo impactó de diversas maneras en el desenvolvimiento del sistema judicial. Alcanzó a sus ámbitos competenciales, innovando en cuanto a las materias de intervención, procesos, roles y sujetos procesales. La entidad del cambio ha sido de tal envergadura que la doctrina más actualizada, siguiendo el pensamiento de Thomas Kuhn (1962), coincidió en sostener que en el derecho de la infancia ha habido, ciertamente, una sustitución de paradigmas.

De manera emblemática y sintética, es posible caracterizar la agonía del paradigma del Patronato -que había regido en la provincia de Buenos Aires en virtud del decreto ley 10067 hasta finales de 1983 - con la desaparición de la figura del juez de menores, así como también de sus equipos profesionales: psicólogos, médicos y asistentes sociales, que como peritos auxiliaban de inmediato en su función jurisdiccional para determinar, en un supuesto dado, la existencia de presunto desamparo moral o material ${ }^{4}$.

Los diversos poderes que por entonces tenía un juez del fuero minoril, fueron fragmentados. Por un

1 Ley sancionada el 29/12/04, promulgada el 14/1/05, publicada en el Boletín Oficial el 27/1/05 y reglamentada por decreto 300/05.

2 Ley del Fuero de Familia y del Fuero Penal del Niño, sancionada el 28/11/06, promulgada el 18/1/07 y publicada en el Boletín Oficial el 2/2/07

3 La Convención Internacional sobre los Derechos del Niño tuvo inicialmente reconocimiento legal en el derecho argentino y posteriormente, a partir de la reforma habida en el año 1994, adquirió jerarquía constitucional (cfr. art. 75 inc. 22 de la Constitución Nacional).

4 En la provincia de Buenos Aires la ideología del Patronato se expresaba a través del decreto ley 10067/83, en el cual se regulaba una amplia competencia del Juez de Menores, ante la denuncia o puesta en conocimiento de que un menor se hallaba en riesgo o desamparo, moral o material. 
lado, se creó un verdadero sistema de justicia penal juvenil orientado a la constatación del hecho punible y a la identificación de sus autores, integrado por jueces de garantías, jueces y/o tribunales de responsabilidad $^{5}$, y por fiscales y defensores oficiales. Por otro, se han reencauzado las demás cuestiones concernientes al reconocimiento de derechos del niño al Fuero de Familia, en donde la figura del Asesor de Incapaces adquiere novísimas implicancias.

Las conflictividades históricamente conocidas como de carácter asistencial, fueron destinadas para su tratamiento al ámbito del Poder Ejecutivo Provincial, a través de sus sistemas locales o zonales de promoción y protección de derechos ${ }^{6}$, en miras a la desjudicialización de las cuestiones de infancias, y en el afán de lograr respuestas inmediatas en el propio seno municipal y comunitario.

Si bien en muchos aspectos la consagración del nuevo sistema en la provincia de Buenos Aires ha sido muy auspiciosa, las graves deficiencias constatadas durante su proceso de implementación, revelaron la existencia de lagunas normativas. Ausencias en la previsión legal que se traducen en lo empírico, en la falta de respuesta del Estado para asumir y disuadir adecuadamente a los niños y jóvenes de la comisión de hechos delictuales. Esto, ciertamente, constituye una severa limitación, que lejos está de coadyuvar a la contención o limitación de los grados de violencia que rigen en una sociedad.

Un tema particularmente espinoso es el relativo a las medidas judiciales que pueden ser aplicadas a los jóvenes que, en un determinado caso, pueden verse incursos en la comisión de un hecho penalmente relevante.

5 El sistema de integración de los Tribunales de Responsabilidad Penal Juvenil ha sido cuestionado por la complejidad que supone su operativización. Las dificultades se advierten por ejemplo en aquellos departamentos judiciales en donde la cantidad de jueces de Responsabilidad Juvenil y jueces de Garantías del Joven resulta insuficiente y ello se torna más complejo si se atiende al traslado de los magistrados que deba realizarse atento las amplias extensiones territoriales de nuestra provincia (art. 27 de la ley 13634).

6 Entre los pilares de este nuevo sistema se hallan la descentralización institucional que responde a la concepción de que las diferentes problemáticas de los niños deberían encontrar respuesta en el ámbito comunitario. En este sentido, fueron modificadas las jurisdicciones administrativas provinciales, que ahora se corresponden con la división territorial del Ministerio de Educación.
En este orden cabe destacar que la ley 13634 prevé como facultad del juez (a pedido de parte) la imposición de medidas cautelares sobre el joven imputable (de 16 a 18 años) y en casos graves como última ratio, también admite que se dicte prisión preventiva, la cual puede durar seis meses y, excepcionalmente, prorrogarse a pedido del fiscal, por seis meses más (cfr. art. 43 de la ley 13634).

Concluida la audiencia de debate o juicio propiamente dicho, el legislador provincial además contempló, con inusitada tensión con la legislación nacional ${ }^{7}$, la imposición por el juez de ciertas consecuencias jurídicas a quien, a la luz de las reglas del debido proceso, haya sido declarado penalmente culpable por la comisión de delito (cfr. arts. 37 y 40 Convención Internacional sobre los Derechos del Niño, entre otros tratados internacionales). Pero, por otra parte también estableció de modo extraño, la posibilidad del juez en aplicar medidas judiciales a los niños no punibles, ya sea por razones de edad (menores de 16 años) o por otras causales que le hayan impedido comprender acabadamente su accionar contrario a derecho (cfr. arts. 64 y 68 de la ley 13634).

Vale la pena detenerse en estas reglas, pues, como puede vislumbrarse, el profundo abordaje de esta problemática excede con creces la mera contemplación del texto legal desde el prisma únicamente judicial; y exige, por tanto, la intervención coordinada de especialistas, expertos en temas de infancia, que desde distintos ámbitos del Estado se encuentren habilitados a trabajar integralmente sobre el joven y su entorno familiar.

A tales efectos, la propia interdisciplinariedad que caracteriza la materia de infancia, impone la necesidad de contar con la organizada y capacitada colaboración de los equipos técnicos de los Servicios Locales y Zonales de Promoción y Protección de Derechos, como así también los que integran el actual Cuerpo Técnico Auxiliar

7 La ley 22278 establece el régimen penal juvenil en la República Argentina, pero si bien se encuentra vigente, expresa los principios de Paradigma del Patronato, por lo que se prevé su próxima reforma a fin de "aggiornar" la legislación nacional al programa político proclamado en la CIDN. 
(Martínez, Del Grosso \& Merlino, 2010), pertenecientes a la Asesoría Pericial de la Suprema Corte de Justicia de la Provincia de Buenos Aires - SCBA. En ocasiones, asimismo se requiere articular la intervención judicial, trasladando la inicial competencia penal por un hecho, hacia al ámbito del Fuero de Familia.

Este delicado entramado organizacional, no se encuentra en la actualidad armoniosamente instaurado en la provincia de Buenos Aires, generándose en la práctica preocupantes disfuncionalidades. A modo de ejemplo, esto ha quedado explicitado en más de una ocasión, cuando un joven no punible comete de manera reiterada conductas delictivas, siendo las intervenciones del Estado previstas por la nueva ley como totalmente inocuas o al menos insuficientes, para desmotivar ese accionar.

En la Tabla 1, se expone la distribución de causas iniciadas en el fuero y se pone de manifiesto una variabilidad muy grande del promedio de causas por juez y por año, lo que indica que también está pendiente ajustar aspectos muy concretos de la organización jurisdiccional, como es el nombramiento de jueces en función de criterios empíricos.

Aún es extenso el camino que resta por transitar para acceder a la definitiva construcción de un sistema judicial capaz de homologar en su materialidad los principios que sustentan el paradigma de la promoción y protección integral de derechos.

A la luz del nuevo paradigma, cuantiosas son las cuestiones por repensar y consensuar, en relación con la debida intervención de la justicia respecto de los jóvenes que efectivamente hayan infringido la ley penal. Pero mayores son los desafíos cuando se comienza a reflexionar acerca de cómo debe desarrollarse la eficaz intervención del Estado en clave preventiva, y en este contexto particular adquiere relevancia el estudio del Derecho Comparado.

Es una verdad a voces, sostener que para combatir la violencia juvenil se necesitan programas

TABLA 1

Distribución de causas iniciadas en el Fuero de Responsabilidad Penal Juvenil

\begin{tabular}{|c|c|c|c|c|}
\hline \multicolumn{5}{|c|}{ Juzgados de Responsabilidad Penal Juvenil. Causas Ingresadas Periodos 2008 y 2009} \\
\hline Departamento Judicial & 2008 & 2009(1) & $\begin{array}{l}\text { Cantidad de } \\
\text { Juzgados }\end{array}$ & $\begin{array}{l}\text { Promedio } \\
\text { por juzgado }\end{array}$ \\
\hline Azul & 17 & 123 & 1 & 123 \\
\hline Bahía Blanca & 18 & 8 & 2 & 4 \\
\hline Dolores & 68 & 195 & 1 & 195 \\
\hline Junín & 1 & 156 & 1 & 156 \\
\hline La Matanza & 14 & 482 & 2 & 41 \\
\hline La Plata & 379 & 239 & 2 & 120 \\
\hline Lomas de Zamora & 54 & 347 & 3 & 116 \\
\hline Mar del Plata & 18 & 221 & 2 & 111 \\
\hline Mercedes & 17 & 117 & 1 & 117 \\
\hline Morón & 38 & 117 & 2 & 59 \\
\hline Necochea & 2 & 13 & 1 & 13 \\
\hline Pergamino & 4 & 67 & 1 & 67 \\
\hline Quilmes & 154 & 130 & 2 & 65 \\
\hline San Isidro & 17 & 117 & 3 & 39 \\
\hline San Martín & 54 & 202 & 2 & 101 \\
\hline San Nicolás & 5 & 63 & 1 & 63 \\
\hline Trenque Lauquen & - & 31 & 1 & 31 \\
\hline Zarate Campana & 5 & 21 & 1 & 21 \\
\hline Total & 870 & 2649 & 29 & 91 \\
\hline
\end{tabular}

Nota. (1) Los datos de 2009 son preliminares.

Fuente: Datos obtenidos de las planillas remitidas por los titulares de los órganos en cumplimiento de las resoluciones de inventario. 
que se implementen antes de que tenga que intervenir el sistema judicial. Programas que no solo aborden al joven en particular, sino también a su familia, a la escuela, al barrio y a la comunidad en que se inserta. Programas en donde asimismo sea posible discriminar a los niños y jóvenes que presenten un determinado nivel de riesgo de caer en tales comportamientos dañosos relevantes penalmente, como así también que diluciden cuáles son los recursos con los que se cuentan y, consiguientemente, cuál sería su más eficaz distribución (Roesch, 2007).

Ahora bien, la evolución de la ciencia ha permitido hallar herramientas metódicas para proceder a tales evaluaciones (Roesch, 2007). Sin embargo, lo que aún queda por dilucidar es, si la provincia de Buenos Aires, en este marco de transición normativa, está a la altura de las circunstancias para lograr desarrollar una política pública integral de tales connotaciones, propias de un estado democrático y de derecho, que se constituya como real garante de la vida pacífica en sociedad.

\section{El Ministerio de Desarrollo Social}

En la provincia de Buenos Aires hay tres tipos de dispositivos penales dependientes de la Subsecretaría de Niñez y Adolescencia del Ministerio de Desarrollo Social: a) los centros cerrados (institutos de seguridad), b) los centros de contención (régimen de semilibertad) y c) los centros de referencia. En estos últimos no se alojan jóvenes, sino que realizan el seguimiento (no control) de las medidas alternativas a la prisión, tales como suspensión de juicio a prueba, tareas comunitarias, arresto domiciliarios, medidas socioeductativas, etc., dispuestas por los jueces del fuero. En la provincia hay veintiún centros de este tipo.

El flujo de los jóvenes hacia esos lugares de alojamiento está precedido por el estadio inicial en los Centros de Recepción. En la provincia de Buenos Aires hay cuatro de estos centros y están distribuidos de manera tal de cubrir amplias y diferentes zonas geográficas. La planificación de este estadio inicial incluía el proceso de evaluación y la derivación a las restantes instituciones según criterios. Los criterios originalmente pensados desde el órgano administrativo tuvieron un sentido subjetivo, es decir, consideraban las características del joven: a) su compromiso con el delito, b) primera causa o reincidente, c) grado de compromiso con las drogas, d) nivel de estudio, e) lugar de residencia a los fines de no producir un desarraigo y posibilitar la vinculación con su familia y f) edad del joven, entre otras. Esta planificación ideal fue paulatinamente virando hacia un sistema más pragmático, que prioriza por ejemplo las características del delito, de tal manera que si el joven está acusado de un delito muy grave como homicidio, se le busca un lugar que imposibilite la fuga. Otro criterio adoptado es el de la adaptación institucional del joven, en el sentido que si tiene conductas muy conflictivas en su convivencia con los pares, se lo ubica en un dispositivo de máxima seguridad independientemente del delito cometido.

Por otra parte, los jueces cuando remiten a los jóvenes frecuentemente disponen si debe ir a un régimen cerrado o semiabierto, por lo que deja poco poder de decisión a la institución administrativa. Esto genera algunas dificultades en la búsqueda más apropiada del lugar de alojamiento.

Por último, en ocasiones las necesidades de urgencia hacen que, por lo menos transitoriamente, los criterios de derivación deban ser postergados en función de tener que optar por el lugar donde se produce una vacante. La saturación del sistema hace, también, que estos centros de recepción funcionen, de hecho, como centros cerrados, pues los jóvenes permanecen en dichos lugares más de lo que requeriría el estadio de evaluación inicial.

Los centros de referencia tienen por objeto y finalidad la atención ambulatoria, para el cumplimiento de medidas cautelares o sancionatorias alternativas a la restricción o privación de la libertad ambulatoria, ordenadas por los tribunales competentes en el marco de un proceso penal seguido a personas menores de edad. Sus funciones son de evaluación, atención y/o derivación de los jóvenes a programas desconcentrados en municipios u organizaciones de la comunidad.

Así, en los centros de referencia ingresan jóvenes de ambos sexos de hasta 18 años edad, para 
cumplir con cualquiera de las medidas contempladas en la ley 13634 (a excepción de la privación de la libertad) y ordenadas por la autoridad judicial competente.

El personal de los centro de referencia lleva a cabo el control de las medidas judiciales ordenadas y, por intermedio de instituciones de la comunidad, brinda asesoramiento, apoyo en ese sentido, acompaña al joven a realizar diversas diligencias personales referidas a su documentación personal, a su inserción en el ámbito educativo formal o en el mercado laboral y a su estado de salud. Asimismo, elabora estrategias de inserción del joven en la comunidad (ya sea a través de los programas oficiales o por intermedio de instituciones de la comunidad) y brinda asesoramiento y apoyo al joven y a su grupo familiar a los fines de elaborar un proyecto de vida digno. Para tales efectos, el referido personal suele concurrir al domicilio del joven para tomar contacto con su grupo familiar, a los establecimientos educativos, deportivos o laborales, entre otras instituciones de la sociedad civil.

En el mes de octubre de 2010, los centros de referencia brindaban servicio a 3.116 jóvenes (Tabla 2). Esta amplia población varía constantemente dado que por diversos motivos, verbigracia, por el cese de la medida u obligación impuesta ya que se dan altas y bajas. Por ejemplo, en el mismo mes se produjeron 111 bajas y 225 altas.

Respecto a los centros de contención, la provincia de Buenos Aires dispone de 14 con una capacidad para 167 jóvenes; al mes de noviembre de 2010 había alojados 111 jóvenes que habían cometido delitos según la siguiente distribución: 35 homicidios, 37 robos calificados, 15 robos agravados, 3 abusos sexuales y el resto en la categoría de otros delitos. El funcionamiento se caracteriza por la semilibertad del joven y la flexibilidad en la implementación de diversos servicios. Entre los servicios se destaca la asistencia para la inserción laboral, la facilitación del acceso al tratamiento ambulatorio o bajo internación en comunidades terapéuticas de trastornos por abuso de sustancias, la derivación a clínicas psiquiátricas o a los centros cerrados. Recientemente, se implementó un nuevo servicio con la denominación de Programa Volver, que consiste en la adjudicación de una beca al joven y de un tutor en el barrio. El programa está destinado a jóvenes y adolescentes de hasta 25 años en conflicto con la ley penal, que se encuentren en procesos de externación o cumpliendo medidas socioeducativas alternativas a la privación de la libertad. El objetivo del programa es favorecer la integración social y las alternativas vitales alejadas del delito de jóvenes pertenecientes a comunidades vulnerables. Cuenta con sedes en el espacio local (municipios) con recursos profesionales, técnicos y logísticos para el desarrollo de estrategias de contención y seguimiento de los jóvenes.

El programa pretende llegar a tener dos equipos técnicos centrales, 30 equipos técnicos distribuidos en diversos municipios del conurbano, 160 profe-

TABLA 2

Jóvenes en Centros de Referencia distribuidos por Departamento Judicial

\begin{tabular}{lc}
\hline \multicolumn{1}{c}{ Localidad del Centro de Referencia } & Octubre \\
\hline Azul & 91 \\
Bahía Blanca & 79 \\
Dolores & 63 \\
Junín & 127 \\
La Matanza & 441 \\
La Plata & 110 \\
Lomas de Zamora & 183 \\
Mar del Plata & 277 \\
Mercedes & 110 \\
Moreno & 313 \\
Morón & 291 \\
Necochea & 47 \\
Pergamino & 65 \\
Quilmes & 130 \\
San Isidro & 228 \\
San Martín & 244 \\
San Nicolás & 77 \\
Tandil & 46 \\
Trenque Lauquen & 105 \\
Tres Arroyos & 25 \\
Zarate/Campana & 64 \\
\hline Total & 3116 \\
\hline Nota. Los Cen & \\
\hline
\end{tabular}

Nota. Los Centros de Referencia tienen una amplia distribución en el territorio de la provincia, intentando de mantener la vigencia de vínculos entre el joven y su entorno primario.

Fuente: elaboración propia. 
sionales e idóneos, 1.000 jóvenes bajo seguimiento. Hasta el momento, el programa alcanza a 550 jóvenes que cobran una beca mensual.

En los centros cerrados, la edad de los jóvenes varía, entre 14 y 21 años. Al mes de noviembre de 2010, había alojados 463 jóvenes. Su distribución por grupo etario era la siguiente: 11 menores de 16 años, 263 entre 16 y 18 años, y 189 mayores de 18 años. La situación legal de la población era la siguiente: 37 detenidos, 329 con prisión preventiva, 19 con medida de seguridad, 30 con sentencia y 48 con condena.

El sistema tiene jóvenes de hasta 21 años porque, habiendo ingresado siendo menores de 18 años, al cumplir la mayoría de edad no son trasladados a unidades carcelarias. Este tema resulta conflictivo porque sobre él confluyen diversas reglamentaciones e interpretaciones. El articulo 6 de la Ley $\mathrm{Na}$ cional 22278 (1980) que establece el Régimen Penal de la Minoridad todavía vigente señala que: "Las penas privativas de libertad que los jueces impusieran a los menores se harán efectivas en institutos especializados. Si en esta situación alcanzaren la mayoría de edad, cumplirán el resto de la condena en establecimientos para adultos". Respecto de este artículo, algunos jueces, en su momento, interpretaban que la mayoría de edad a la que se hace referencia es a la mayoría de edad civil, que era 21 años, y otros entendían que se refería a la mayoría de edad penal. De esta manera, algunos jueces disponían el alojamiento en unidades carcelarias y otros no. El 22 de diciembre de 2009, entró en vigencia la ley 26579, que en su artículo 1ํㅡㄹ modifica el artículo 126 del Código Civil, estableciendo la mayoría de edad civil a los 18 años, por ende no deberían persistir mayores discusiones al respecto; sin embargo, se abrieron nuevas alternativas de interpretación respecto al establecimiento para adultos al que hace referencia el artículo. Las alternativas se refieren a si el establecimiento debe depender del Ministerio de Desarrollo Social o si puede ser uno dependiente del Ministerio de Justicia, es decir, una unidad carcelaria. Si la interpretación es la primera, el juez dispone que el joven permanezca en estos establecimientos. En contra de esta interpretación, el artículo 15 de la Ley de Ejecución Penal de la provincia de Bue- nos Aires (1999) establece que los jóvenes adultos de 18 a 21 años serán alojados en establecimientos o secciones especiales con el objeto de facilitar el desarrollo de aquellos programas asistenciales y/o de tratamiento que, implementados para pequeños grupos, contemplen con especial énfasis los aspectos formativo educativos de los mismos, teniendo en cuenta la especificidad de los requerimientos propios de la edad. En el mismo sentido, apoya el artículo 85 de la ley 13634 (2006) que rige el Sistema Penal Juvenil de la Provincia de Buenos Aires, que impone que será de aplicación subsidiaria la legislación provincial sobre ejecución de penas o de medidas impuestas a los procesados, en la medida en que no restrinja los derechos reconocidos por la presente ley.

Los centros cerrados brindan servicios educativos de diversas características y con variantes entre centros: escuela, taller de comunicación social, taller de carpintería, fábrica de pastas, fábrica de bloques de ladrillo, taller cultural de video, tareas gastronómicas en la cocina de la institución -con remuneración-, actividades gimnásticas, actividades de mantenimiento de la institución. Por otra parte, tienen acceso a tratamiento psicológico y psiquiátrico según demanda o necesidad. En estos centros se destaca la intervención del Servicio de Trabajo Social que ejerce una tarea de enlace entre los diferentes servicios, el joven, su familia y el sistema judicial, para el que emiten informes frecuentemente.

El personal directivo no es profesional, con la excepción de uno de los centros que cuenta con un psicólogo en el cargo de director. En general, está conformado por asistentes de minoridad. Los profesionales de salud mental suelen brindar servicios en más de una institución Todos los centros tiene psicólogo; los psiquiatras brindan servicio en más de una institución. En términos generales, hacen una entrevista inicial y luego actúan en función de la demanda del joven o del personal que se denomina efectivo. Los relatos dan cuenta dos roles diferenciados entre los profesionales y el personal efectivo; mientras los últimos realizan actividades más ejecutivas y con contacto más estrecho con el joven, los primeros ejercen función más esporádica 
y en respuesta a eventuales necesidades. Si bien se podría considerar que este tipo de división del trabajo es esperable en función de la distribución del tiempo laboral, produce también una percepción de falta de compromiso en el ejercicio profesional y que se carezca de una planificación técnica integral de la intervención. Paralelamente a la descripción de este distanciamiento de los sectores del plantel, se argumenta legitimándolo con base en que no es imprescindible la intervención regular del profesional de la salud mental, pues los jóvenes necesitan una aproximación normal de los servicios.

Por otra parte, es importante señalar algunas consecuencias de la transición de sistemas. Uno de los problemas emergentes consistió en críticas recibidas por los profesionales que, acostumbrados a incluir en sus informes aspectos relacionados con la causa penal, transmitían información que resultaba contraproducente para las acciones de la defensa en juicio. Frases comunes de los informes profesionales como "se arrepintió de lo que hizo" resultaban en una criticable transferencia de información desde el mundo privado al mundo controversial del juicio.

A continuación se exponen estadísticos descriptivos del flujo de jóvenes ingresados a las instituciones en el marco de un proceso penal.

Durante el año 2010, ingresaron 1.463 jóvenes (98\% varones) a las diversas instituciones propias del Ministerio de Desarrollo Social o relacionadas

TABLA 3

Distribución de jóvenes por tipo de institución

\begin{tabular}{lcc}
\hline \multicolumn{1}{c}{ Tipo de Institución } & $\begin{array}{c}\text { Nuevos } \\
\text { ingresos }\end{array}$ & $\begin{array}{c}\text { Egresos sin } \\
\text { consentimiento }\end{array}$ \\
\hline Centro Cerrado & 414 & 28 \\
Centro de Contención & 143 & 116 \\
Centro de Recepción & 870 & 27 \\
Comunidades Terapéuticas & 34 & 51 \\
Clínicas Psiquiátricas & 2 & 2 \\
\hline Total & 1463 & 224 \\
\hline
\end{tabular}

Nota. Se destaca el alto número de egresos sin consentimiento en los centros de intervención ambulatoria y en las Comunidades Terapéuticas para jóvenes con dependencia a drogas.

Fuente: elaboración propia. con este a través de convenios clínicas o comunidades terapéuticas. De todos los alojados, independientemente del año en que ingresaran, egresaron con consentimiento judicial 980 y sin consentimiento 224 (Tabla 3).

La distribución por edad pone de manifiesto que las mayores frecuencias se hallaron a los 17, 16 y 15 años de edad (Tabla 4).

Existe una importante movilización de jóvenes entre una institución y la otra. Además de aquella movilización que se realiza con base en la lógica del programa (desde el centro de recepción a los restantes), se producen otras movilizaciones no sistemáticas producto de órdenes judiciales, emergencias, situación familiar, etc. Una amplia proporción de los jóvenes permanece alojado en las dependencias menos de 60 días. La distribución de estadías adquiere, en general, la forma de la de los Centros Cerrados (Gráfico 1). La excepción la constituyen las clínicas psiquiátricas, en las que suelen internarse pocos jóvenes que tienen características bien diferentes: aquellos con problemas de salud que demandan cortas intervenciones y aquellos con problemas crónicos con prolongadas internaciones (Gráfico 2).

TABLA 4

Distribución de los jóvenes por edad al ingreso

\begin{tabular}{|c|c|}
\hline Edad en años & $\mathrm{N}$ \\
\hline 12 & 3 \\
\hline 13 & 12 \\
\hline 14 & 40 \\
\hline 15 & 118 \\
\hline 16 & 509 \\
\hline 17 & 728 \\
\hline 18 & 46 \\
\hline 19 & 4 \\
\hline 20 & 3 \\
\hline Total & 1463 \\
\hline
\end{tabular}

Nota. Jóvenes de ambos sexos.

Fuente: elaboración propia. 


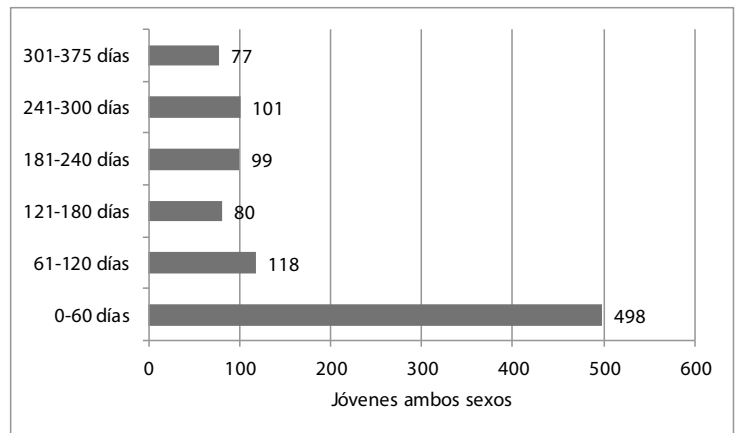

Gráfico 1. Distribución de los jóvenes institucionalizados según duración de la estadía.

Nota. Estadías en Centro Cerrados de la provincia de Buenos Aires (período 2010).

Fuente: elaboración propia.

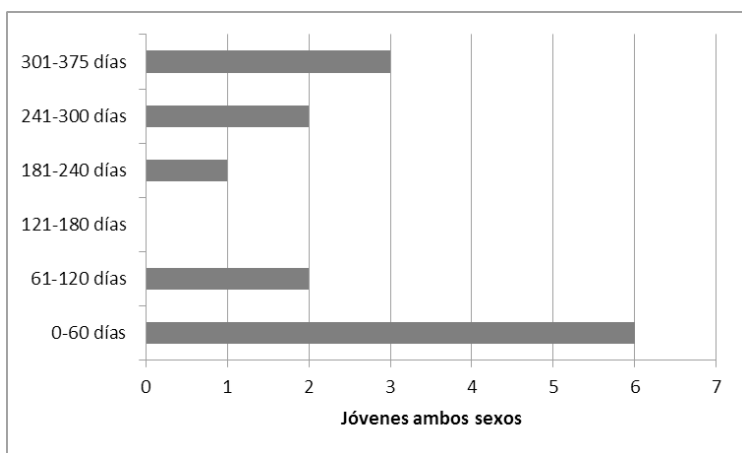

Gráfico 2. Distribución de los jóvenes internados según duración de la estadía.

Nota. Estadías en clínicas psiquiátricas de la Provincia de Buenos Aires (período 2010).

Fuente: elaboración propia.

\section{La evaluación del joven que toma contacto con la justicia juvenil}

El joven que ha cometido algún delito entra en contacto con expertos en salud mental en diferentes contextos y por diferentes cuestiones. Puede ser evaluado para contribuir a la toma de decisión judicial relacionada con la imputabilidad o el riesgo de nuevos delitos y para establecer qué servicios de salud mental requiere. Aún sin haber sido procesado, cuando presenta conductas riesgosas también puede ser evaluado a los efectos de planificar prevención desde el entorno asistencial (Antoniu et al., 2010; Bradshaw, Schaeffer, Petras \& Ialongo, 2010).
La evaluación desde la salud mental no es un fin en sí mismo, sino un paso imprescindible, si es que se pretende generar una intervención que respete el derecho a la salud del joven, que se ajuste a sus necesidades y que persiga el desarrollo saludable y autónomo del joven. Su complejidad impone una perspectiva técnica sumamente amplia que explore la multifactoriedad interviniente. A continuación se comentan recientes hallazgos que enriquecen la evaluación de esos múltiples factores.

\section{Evaluación de factores familiares y contextuales}

La evaluación del joven no se restringe a sus características personales. Por el contrario, por no haber completado el tránsito hacia la adultez, resulta imprescindible revisar los nexos de relación y las influencias ecoculturales vigentes. Una evaluación integral y compleja como la mencionada puede ilustrarse de hallazgos de investigaciones cualitativas que ponen de manifiesto aspectos procesales de las influencias familiares y de pares. En esta línea, son tradicionalmente reconocidas influencias desfavorables de familias anómicas o autoritarias. Martín, Martínez y Rosa (2009) también agregan las influencias que generan familias denominadas bipolares, y que están caracterizadas por que a medio o largo plazo modifican de manera extrema sus pautas educativas y socializadoras. En general, se trata de familias que al principio son más claramente anómicas, pero que cuando deben afrontar consecuencias fuertemente negativas de la conducta de sus hijos, por ejemplo, ante denuncia policial o heridas que requieren hospitalización o atención médica de urgencia, reaccionan abruptamente como una familia autoritaria, con castigos, restricciones del ocio y amenazas.

Las familias pueden aportar factores influyentes de importancia para el riesgo de las conductas externalizadas. Recientemente, Buschgens et al. (2010) realizaron un estudio con 2.230 preadolescentes de 10 a 12 años que participan de un estudio de cohorte en Holanda y hallaron que, en general, los preadolescentes que percibieron carencia de calidez emocional en el estilo parental y alto nive- 
les de rechazo o sobreprotección fueron descriptos por sus padres y maestros como menos atentos, con más agresiones y actos delictivos. Este tipo de información de historia familiar se puede facilitar introduciendo viñetas de las diversas condiciones psicopatológicas.

\section{Evaluación de personalidad y de trastornos psíquicos}

La evaluación para detectar trastornos psíquicos y características de personalidad que permitan el mejor ajuste a la intervención debería ser una acción rutinaria. Algunos instrumentos resaltan por su amplia utilización. Baum, Archer, Forbey y Handel (2009) hicieron una amplia revisión de la literatura producida entre 1992 y 2007 con el objetivo de evaluar en qué medida se ha investigado con el Minnesota Multiphasic Personality Inventory-Adolescent (MMPI-A) y del Millon Adolescent Clinical Inventory (MACI) en muestras obtenidas del ámbito de la justicia juvenil, y la fuerza de la asociación entre sus escalas y diversos criterios relevantes. Los autores informan que el área forense fue la que concentró la mayor frecuencia de estudios del MACI y la segunda mayor frecuencia de estudios con el MMPI-A. También aportan información del perfil promedio obtenido con ambos instrumentos en numerosas muestras, por ejemplo, características elevaciones de escalas básicas del MMPI (desvío psicopático, paranoia y hipomanía), de escalas de contenido (trastornos de conducta, problemas escolares, indicadores negativos de tratamiento). También elevaciones de las escalas: transgresor, oposicionista, insensibilidad social, discordia familiar y predisposición delictual del MACI. Resulta interesante que el MACI también tenga respaldo empírico en Chile, donde un estudio permitió sostener la capacidad del instrumento para discriminar entre adolescentes mujeres infractoras de la ley y adolescentes del ámbito clínico (Vinet \& Alarcón, 2009).

Está ampliamente difundido que es importante profundizar en la evaluación de rasgos psicopáticos, dada sus consecuencias devastadoras en múltiples áreas. En Latinoamérica, recientes estudios con las versiones argentina y chilena de la PCL-YV orientan a sostener su confiabilidad y la validez concurrente con escalas del MACI y otras medidas de impulsividad y trastornos de conducta (Folino, 2005; Folino et al., 2009; Zúñiga, Vinet \& León, 2011).

En el mismo sentido, hay un creciente interés en el estudio de potenciales precursores de psicopatía debido a su importancia en la comprensión del proceso evolutivo de la psicopatía y en la prevención (Frick, 2009). Sobre el tema, resulta valiosa la identificación de un grupo de rasgos de crueldad y frialdad afectiva que caracteriza a un subgrupo de jóvenes con características antisociales, aumento de riesgo de violencia y pobre respuesta al tratamiento. En la evaluación de estos rasgos, recientemente se revisaron la validez y confiabilidad del Inventory of Callous and Unemotional Traits (ICU) tanto en su versión auto como heteroinformada (Roose, Bijttebier, Decoene, Claes \& Frick, 2010). Los resultados orientan a considerar al ICU como un promisorio instrumento para la evaluación de los rasgos de crueldad y frialdad afectiva en jóvenes.

Un aspecto más sutil, el coraje en niños y adolescentes, es una condición importante de evaluar. El coraje, además de poder interferir con el desarrollo del miedo patológico y de la ansiedad, debería ser diferenciado de la potencialidad que tienen algunos de aproximarse a estímulos peligrosos sin sentir temor. Recientemente, se exploró una medida del coraje en niños y adolescentes (rango 8-13 años), el Courage Measure for Children [CM-C] (Muris, Mayer \& Schubert, 2010). Los resultados sostienen su confiabilidad y aportan a la validez, en tanto pusieron de manifiesto una relación positiva con el rasgo de extraversión y de masculinidad, y una relación negativa con los síntomas de ansiedad.

\section{Riesgo de recidiva delictiva}

El riesgo de recidiva delictiva es un aspecto relevante que debe ser evaluado, pues del mismo deberían depender diversas medidas preventivas. Ratificando que en los jóvenes resulta de suma importancia considerar factores más allá de la personalidad Mulder, Brand, Bullens y Van Marle 
(2010) pusieron de manifiesto recientemente que la conducta antisocial durante el tratamiento, los problemas familiares y los trastornos mentales del Eje I se asociaron con recidiva severa. Los autores destacan que los delincuentes juveniles no son una población homogénea y la consideración de los subgrupos puede ofrecer la mejor información para el manejo del riesgo de aquellos que tienden a ser recidivantes severos. Para la evaluación, utilizaron el Juvenile Forensic Profile, instrumento de 70 ítems desarrollado previamente para investigación, utilizando datos de los archivos.

La evaluación minuciosa de los Trastornos de Conducta también ofrece información valiosa (Bradshaw et al., 2010). Algunos subtipos homogéneos de trastornos de conducta en la temprana adolescencia pueden ser útiles a los propósitos de delimitar etiología y planificar tratamiento y prevención. Lacourse et al. (2010) estudiaron una muestra de tres cohortes de jóvenes de 12 y 13 años a nivel nacional en Canadá $(N=4.125)$ y hallaron 4 grupos: a) sin trastornos de conducta (82.4 \%), b) trastornos de conducta no agresivos (13.9\%), c) trastornos de conducta físicamente agresivos ( $2.3 \%)$ y c) trastornos de conducta mixtos severos (1.4 \%). El grupo trastornos de conducta mixtos severos fue por mucho el que tuvo más probabilidad de eventos desviados y de estilo de vida delictivo. El estudio también resalta la importancia de considerar la severidad de los trastornos con base en conteo de síntomas; los niños que tenían entre 2 y 5 síntomas tuvieron más probabilidades de ser clasificados en el grupo de severidad moderada no agresiva y podrían beneficiarse mucho con programas de prevención e intervención. Los niños que tenían 6 o más síntomas fueron considerados del grupo de trastorno de conducta severo.

\section{Riesgo de recidiva sexual}

La vida sexual es un área de particular importancia en los jóvenes. Recientes estudios informan tasas mensuales de recidiva más altas que los adultos (Caldwell, 2010). También se reconoce que los jóvenes pueden sufrir o cometer actos violentos en sus citas amorosas con consiguien- tes perjuicios en su funcionamiento psicosocial y en el desencadenamiento de trastornos psíquicos (Brown et al., 2009).

\section{Abuso de sustancia}

Los adolescentes involucrados en delitos se introducen en conductas sexuales riesgosas relacionadas con abuso de alcohol que pueden reducirse con intervenciones diseñadas desde la teoría (Bryan, Schmiege \& Broaddus, 2009), lo que indica que, por lo menos, determinados objetivos pueden ser alcanzados con intervenciones apropiadas.

\section{Aportes a la evaluación desde estudios en población general}

Los estudios en comunidad pueden ser orientadores en la tarea de la evaluación individual. Sullivan, Childs y O'Connell (2010) utilizaron una encuesta en una amplia muestra norteamericana que permitió definir cuatro clases latentes: a) grupo que no se introduce en conductas sexuales de riesgo, pero con alta probabilidad de otras conductas de riesgo (5\%); b) grupo de sujetos que se abstienen de conductas de riesgo (36\%); c) otro que se introduce en algunas conductas de riesgo, especialmente actividad sexual, al que denominaron grupo de experimentadores ( $36 \%$ y c) un grupo que de alto y diverso riesgo (22\%) con altas probabilidades de todo tipo de conductas de riesgo. El estudio orienta la estrategia general de selección. Por ejemplo, los experimentadores, dada la poca probabilidad de uso de preservativo y contacto con múltiples parejas, tienen mayor riesgo para las enfermedades de transmisión sexual que los otros grupos. La identificación de un grupo de alto y diverso riesgo posibilitaría concentrar recursos para prevenir conductas problemáticas en varias dimensiones.

\section{Información faltante}

Así como diversos estudios ponen a disposición criterios útiles para la evaluación y tratamiento de los jóvenes, aún resta obtener información que, por lo menos teóricamente, se ha considerado importante. 
Muchos estudios documentan una relación consistente y coherente entre la integración de una pandilla y el aumento de la delincuencia. Una teoría de la participación en una pandilla, que se basa en las teorías de la anomia y de la presión, propone que la pandilla proporciona un medio de satisfacer las necesidades económicas de los jóvenes excluidos de los mercados laborales legítimos. La búsqueda de oportunidades es una estrategia de prevención de pandillas que se basa en esta teoría y en el principio que sostiene que la búsqueda de oportunidades educativas y laborales puede reducir la posibilidad de que los jóvenes formen parte de pandillas. Las técnicas frecuentes dentro de la búsqueda de oportunidades incluyen la instrucción, la educación correctiva, el entrenamiento laboral y la oferta de empleo. Sin embargo, una reciente revisión no encontró pruebas a partir de ensayos controlados aleatorios o ensayos controlados cuasialeatorios de la efectividad de la búsqueda de oportunidades para la prevención de pandillas (Fisher, Montgomery \& Gardner, 2008).

\section{Discusión}

En este texto se han comentado aspectos jurídicos y administrativos del sistema de Justicia Juvenil de la Provincia de Buenos Aires (Argentina) y aportes internacionales recientes de los estudios sobre evaluación de jóvenes que comenten delitos.

Los casos del estudio de Folino et al. (2009) representaban el fracaso de la familia, del sistema educativo y de otras instancias del Estado responsables de la vida de los niños. Tales fallas ocurrían en un contexto que ha expuesto a los jóvenes a diversos desbalances relacionados con el delito (Folino et al., 2004).

Actualmente, un nuevo sistema se va moldeando con limitaciones prácticas pero intentando jerarquizar valores muy caros para la comunidad, como son los derechos del niño. Por otra parte, van produciéndose progresos en las disciplinas de la salud mental que ponen a disposición de los operadores confiables criterios de evaluación, tanto de los jóvenes como del sistema.
Sin embargo, a pesar de los pasos adelante, puede estar ocurriendo que la marcha no sea tan consistente y rápida como se pretende. Puede estar ocurriendo que péndulos ideológicos no permitan encontrar la justa acción, y puede estar ocurriendo que las herramientas disponibles no se estén utilizando en su plenitud.

Cualquier hora es siempre apta para dejar atrás restricciones a falsas alternativas. Ya es tiempo de dejar de plantear caricaturescas dicotomías entre institucionalización y no institucionalización y de abandonar ingenuas creencias que sin intervención los problemas se diluirán.

La mirada al sistema local y a los aportes técnicos orienta a sostener que es necesario un mayor esfuerzo para ampliar y tecnificar la gama de servicios de intervención del Estado tendiente a interrumpir la concatenación entre vulnerabilidad infantojuvenil y delito.

Respecto a la sistemática de evaluación de los jóvenes, cabe reflexionar si las prácticas están a la altura del desarrollo científico. Actualmente, diversos aportes internacionales, incluyendo algunos latinoamericanos, han puesto de manifiesto factores relacionados con los trastornos de conducta y con trayectorias de cronicidad en las dificultades adaptativas; igualmente, tácticas que permiten identificar la amplia variedad de configuraciones personales en la adolescencia que, indudablemente, no pueden ser homogeneizadas a la hora de brindarles el servicio que facilite su mejor desarrollo (Folino \& León Mayer, 2011). Baste para ilustrar los estereotipos que postergan el aprovechamiento de los aportes científicos, el planteo arriba mencionado de que no es imprescindible la intervención del profesional de la salud mental pues los jóvenes necesitan una aproximación "normal" de los servicios. Claro está que resultaría completamente equivocado intervenir con igual tratamiento a quien padece temprana esquizofrenia y a quien, lejos de padecer alguna psicosis, se moviliza con conductas transgresoras de las normas sociales. Lejos están las ciencias de la salud mental de proponer algo semejante; por el contrario, existe un amplio consenso, y la revisión de los recientes hallazgos lo demuestra, de buscar 
la más precisa evaluación y la más específica intervención. Por otra parte, la inclusión de los problemas que tienen los jóvenes en conflicto con la ley en una representación de normalidad resulta una subestimación de las consecuencias tremendas que la mayor parte padece a lo largo de sus vidas: a) recidiva en el crimen, b) mayor deterioro por abuso de sustancias, c) creciente marginalidad y d) mayores probabilidades de muerte, entre otras.

Las ciencias relacionadas con la salud mental y con el comportamiento tienen la potencialidad de contribuir diseñando e implementado múltiples servicios aptos para los diferentes problemas, pero parece que hay una distancia importante entre las potencialidades del estado actual del conocimiento y la implementación. El camino apropiado se establece lejos de los preconceptos. Resalta como conveniente aprovechar lo mejor que la técnica puede brindar y revisar críticamente las acciones de manera tal que no solo se implemente lo óptimo, sino que pueda ajustarse en el tiempo.

Párrafo aparte merecen los inconvenientes vinculados con las críticas a expresiones de los informes profesionales. Estos no son productos exclusivos de la transición entre sistemas. La transición pudo haberlos puesto de manifiesto en el ámbito juvenil, pero en realidad, precedían a los cambios y se basaban en ambigüedades de los roles profesionales que generaban riesgo de fallas en la confidencialidad, entre otros problemas técnicos, éticos y legales. Estas ambigüedades o problemas de doble agencia, como también suele denominárselos, existieron en la administración de justicia tanto para los menores como para los adultos. Años atrás, los profesionales del Servicio Penitenciario solían realizar informes respondiendo a preguntas judiciales acerca de los condenados o sobreseídos por inimputabilidad que ellos mismos asistían (Folino, Vásquez \& Sarmiento, 2000) y en los antiguos Tribunales de Menores trabajaba un equipo técnico que superponía acciones periciales con otras asistenciales y de asesoramiento. En el ámbito penal de adultos este tipo de práctica fue restringiéndose y el sistema de justicia juvenil actual, con sus características contradictorias, también influirá hacia desaparición de tales prácticas. Indudablemente, el Estado debe acompañar el proceso con la inversión para tener el personal suficiente que pueda desempeñar los diversos roles necesarios.

De lo expuesto se colige que en el tránsito a la plena vigencia de un sistema de justicia adecuado a un paradigma de promoción y protección de derechos como el que declama la Convención Internacional sobre los Derechos del Niño, la provincia de Buenos Aires ha hecho avances, pero aún tiene que revisar y prever ciertas modificaciones legales y técnicas que le permitan optimizar su política de infancia.

\section{Referencias}

Antonius, D., Fuchs, L., Herbert, F., Kwon, J., Fried, J. L., Burton, P. R., et al. (2010). Psychiatric assessment of aggressive patients: A violent attack on a resident. American Journal of Psychiatry, 167(3), 253-259. doi: 167/3/253[pii]10.1176/appi. ajp.2009.09010063

Baum, L. J., Archer, R. P., Forbey, J. D. \& Handel, R. W. (2009). A review of the Minnesota Multiphasic Personality Inventory-Adolescent (MMPI-A) and the Millon Adolescent Clinical Inventory (MACI) with an emphasis on juvenile justice samples. Assessment, 16(4), 384-400. doi: 1073191109338264 [pii] 10.1177/1073191109338264

Bradshaw, C. P., Schaeffer, C. M., Petras, H. \& Ialongo, N. (2010). Predicting negative life outcomes from early aggressive-disruptive behavior trajectories: Gender differences in maladaptation across life domains. Journal of Youth and Adolescence, 39(8), 953-966. doi: 10.1007/s10964-009-9442-8

Brown, A., Cosgrave, E., Killackey, E., Purcell, R., Buckby, J. \& Yung, A. R. (2009). The longitudinal association of adolescent dating violence with psychiatric disorders and functioning. Journal of Interpersonal Violence, 24(12), 1964-1979. doi: 0886260508327700 [pii]10.1177/0886260508327700

Bryan, A. D., Schmiege, S. J. \& Broaddus, M. R. (2009). HIV risk reduction among detained adolescents: A randomized, controlled trial. Pediatrics, 124(6), e1180-1188. doi: peds.2009-0679 [pii] 10.1542/ peds.2009-0679 
Buschgens, C. J., van Aken, M. A., Swinkels, S. H., Ormel, J., Verhulst, F. C. \& Buitelaar, J. K. (2010). Externalizing behaviors in preadolescents: Familial risk to externalizing behaviors and perceived parenting styles. European Child \& Adolescent Psychiatry, 19(7), 567-575. doi: 10.1007/s00787009-0086-8

Caldwell, M. F. (2010). Study characteristics and recidivism base rates in juvenile sex offender recidivism. International Journal of Offender Therapy and Comparative Criminology, 54(2), 197 212. doi: 0306624X08330016[pii]10.1177/030662 4X08330016

Fisher, H., Montgomery, P. \& Gardner, F. E. (2008). Opportunities provision for preventing youth gang involvement for children and young people. Cochrane Database of Systematic Reviews, 2(Art. № CD007002), 7-16. doi: 10.1002/14651858. CD007002.pub2

Folino, J. O. (2005). Risk assessment and violent recidivism risk management in convicts from Argentina. In T. Youn (Ed. de la serie) \& Stephanie W. Hartwell (Ed. del volumen), The organizational response to persons with mental illness involved with the Criminal Justice System: Vol. 12. Research in Social Problems and Public Policy (pp. 75-88). Bingley, UK: Emerald Group Publishing Limited. doi: 10.1016/ S0196-1152(05)12004-3

Folino, J. O., Urrutia, M. I., Crivos, M., Tevez, L., Avalos, A., Marchioni, M., et al. (2004). Homicides and socio-economic influences. Jornal Brasileiro de Psiquiatria, 53(3), 175-182.

Folino, J. O., Domenech, E., Gutiérrez, M. A. \& Lescano, M. J. (2009). Delincuencia infantojuvenil y sistema judicial en la Provincia de Buenos Aires, Argentina. Vertex, 20(83), 26-34.

Folino, J. O. \& León Mayer, E. (2011). Juvenile offenders assessment. Current Opinion in Psychiatry, 24(5), 436-441.

Folino, J. O., Vázquez, J. M. \& Sarmiento, D. (2000). Forensic psychiatric system in the Province of Buenos Aires. International Journal of Law and Psychiatry, 23(5-6), 567-577. doi: S0160-2527(00)00060-1 [pii]

Frick, P. J. (2009). Extending the construct of psychopathy to youth: Implications for understanding, diagnosing, and treating antisocial children and adolescents. Canadian Journal of Psychiatry, 54(12), 803-812.

Kuhn, T. (1962). La estructura de las revoluciones cientificas. Chicago: University of Chicago Library.

Lacourse, E., Baillargeon, R., Dupere, V., Vitaro, F., Romano, E. \& Tremblay, R. (2010). Two-year predictive validity of conduct disorder subtypes in early adolescence: A latent class analysis of a Canadian longitudinal sample. Journal of Child Psychology and Psychiatry, 51(12), 1386-1394. doi: JCPP2291 [pii] 10.1111/j.1469-7610.2010.02291.x

Ley Nacional 22278 de 1980. Régimen Penal de la Minoridad. Boletín Oficial de la República Argentina. Agosto 28 de 1980.

Ley de la Provincia de Buenos Aires 12256 de 1999. Ley de Ejecución Penal Bonaerense. Boletín Oficial de la Provincia de Buenos Aires, Suplemento. Enero 25 de 99.

Ley de la Provincia de Buenos Aires 13634 de 2006. Ley del Fuero de Familia y del Fuero Penal del Niño. Boletín Oficial de la Provincia de Buenos Aires. Febrero 2 de 2007.

Ley Nacional 26579 de 2009. Ley modificatoria del Art. 126 del Código Civil Argentino. Boletín Oficial de la República Argentina. Diciembre 22 de 2009.

Martín, M. J., Martínez, J. M. \& Rosa, A. (2009). Las bandas juveniles violentas de Madrid: su socialización y aculturación. Revista Panamericana de Salud Pública, 26(2), 128-36.

Martínez, V., Del Grosso, A. \& Merlino, A. (2010). Consideraciones interdisciplinarias acerca de los adolescentes para la implementación de prácticas alternativas a la privación de libertad. En Secretaria de Derechos Humanos Ministerio de Justicia, Seguridad y Derechos Humanos de la Nación (Ed.), Justicia restaurativa en el sistema de responsabilidad penal juvenil: conceptos, perspectivas y mecanismos procesales para su implementación (pp. 11-23). Buenos Aires: República Argentina, Presidencia de la Nación.

Mulder, E., Brand, E., Bullens, R. \& Van Marle, H. (2010). A classification of risk factors in serious juvenile offenders and the relation between patterns of risk factors and recidivism. Criminal Behaviour and Mental Health, 20(1), 23-38. doi: 10.1002/ cbm.754 
Muris, P., Mayer, B. \& Schubert, T. (2010). "You might belong in Gryffindor": Children's courage and its relationships to anxiety symptoms, big five personality traits, and sex roles. Child Psychiatry \& Human Development, 41(2), 204-213. doi: 10.1007/ s10578-009-0161-x

Roesch, R. (2007). Delincuencia juvenil, riesgos y prevención. En J. M. Sabucedo \& J. Sanmartín (Coords.), Los escenarios de la violencia (pp. 215 232). Barcelona: Ariel.

Roose, A., Bijttebier, P., Decoene, S., Claes, L. \& Frick, P. J. (2010). Assessing the affective features of psychopathy in adolescence: A further validation of the inventory of callous and unemotional traits.
Assessment, 17(1), 44-57. doi: 1073191109344153 [pii]10.1177/1073191109344153

Sullivan, C. J., Childs, K. K. \& O'Connell, D. (2010). Adolescent risk behavior subgroups: An empirical assessment. Journal of Youth and Adolescence, 39(5), 541-562. doi: 10.1007/s10964-009-9445-5

Vinet, E. \& Alarcón, P. (2009). Caracterización de personalidad de mujeres adolescentes infractoras de ley: un estudio comparativo. Paideia, 19(43), $143-152$

Zúñiga, D., Vinet, E. \& León, E. (2011). Caracterización psicométrica del Psychopathy Checklist: Youth Version (PCL:YV) en adolescentes chilenos. Terapia Psicológica, 29(1), 25-31. 
